\title{
Positive solutions of a weakly singular periodic eco-economic system with changing-sign perturbation
}

\author{
Teng Ren ${ }^{\mathrm{a}, *}$, Sidi Li ${ }^{\mathrm{b}}$, Xinguang Zhang ${ }^{\mathrm{c}, \mathrm{d}}$ \\ a School of Logistics and Transportation, Central South University of Forestry and Technology, Changsha 410004, China. \\ ${ }^{b}$ School of Tourism Management, Central South University of Forestry and Technology, Changsha 410004, China. \\ ${ }^{c}$ School of Mathematical and Informational Sciences, Yantai University, Yantai 264005, Shandong, China. \\ ${ }^{d}$ Department of Mathematics and Statistics, Curtin University of Technology, Perth, WA 6845, Australia.
}

Communicated by A. Meskhi

\begin{abstract}
In this paper, we establish the positive bounded solutions for a changing-sign periodic perturbed differential system with weak singularity in eco-economic and other applied fields. The conditions for the existence of solution are established for the positive, negative and semipositone cases of nonlinear term, and the perturbation is allowed to be a singular and changing-sign $\mathrm{L}^{1}(0, \mathrm{~T})$ function. (C)2017 All rights reserved.
\end{abstract}

Keywords: Weak singularity, bounded solutions, sign-changing perturbation, periodic problems, eco-economical system. 2010 MSC: 34B15, 34B18.

\section{Introduction}

This paper is motivated by the following changing-sign periodic perturbed eco-economic system

$$
\left\{\begin{array}{l}
u^{\prime \prime}(t)+u(t)=\frac{3}{2 v^{\frac{1}{2}}(t)}-\frac{t^{-\frac{1}{2}}}{100}, \quad v^{\prime \prime}(t)+v(t)=u^{2}(t), \\
u(0)=u(1), \quad u^{\prime}(0)=u^{\prime}(1), \quad v(0)=v(1), \quad v^{\prime}(0)=v^{\prime}(1),
\end{array}\right.
$$

which arises naturally in eco-economic and noise or stochastic perturbation theory $[14,15,17,21]$ as well as in most of man-made phenomena $[11,20]$ and the field of applied mathematics $[4,7,10,12,16,22,23$, 25-29]. In perturbation model (1.1), the forcing perturbed function $-\frac{t^{-\frac{1}{2}}}{100}$ often plays an important role for whole system, thus it is necessary to study the influence of perturbed function in order to get an in-depth understanding for the model system.

\footnotetext{
${ }^{*}$ Corresponding author

Email addresses: chinarenteng@163.com, hunantengren@163.com (Teng Ren), sidilihunan@163.com (Sidi Li), zxg123242@163.com (Xinguang Zhang)

doi:10.22436/jnsa.010.05.22
} 
Recently, Ward [26] studied the existence and non-existence of T-periodic solutions for T-periodic nonlinear differential equation with a forcing perturbed function $e(t) \in C([0, T], \mathbb{R})$

$$
\left\{\begin{array}{l}
u^{\prime \prime}(t)+g(u(t))=e(t) \\
u(0)=u(T), \quad u^{\prime}(0)=u^{\prime}(T) .
\end{array}\right.
$$

By using the variational and topological methods, the existence and non-existence results for the equation (1.2) were obtained provided that the bounded nonlinear term $g$ satisfies $g(s)>0$ (or $g(s)<0$ ) for all real numbers $s$ and $g(\infty)=0$. Clearly, if the forcing function $e(t)$ is defined on the entire real line and is T-periodic then any solution to (1.2) can be extended to the whole real line as a T-periodic solution of (1.2). The work of James and Jr differs from most of existing work which relates to nonlinearities $g(u)$ such that either

(i) $g(u) u \geqslant 0$ for $|u|$ large; or

(ii) $g(u)$ is periodic with zero mean, or $g(u)$ is oscillatory.

However, we also notice that $e(t) \in \mathrm{C}([0, \mathrm{~T}], \mathbb{R})$ and $g(t)$ is bounded continuous function satisfying $g( \pm \infty)=0$ in [26]. So a natural problem is if forcing perturbed function is unbounded (even is singular) such as $e(t) \in \mathrm{L}^{1}((0, T), \mathbb{R})$ and $\mathrm{g}(+\infty)=+\infty$, what results should exist?

Base on the above fact, the purpose of this paper is to study the following general changing-sign periodic perturbed differential system which covers equations (1.1) and (1.2) as special case

$$
\left\{\begin{array}{l}
u^{\prime \prime}(t)+a(t) u(t)=f(t, v(t))+e(t), \quad v^{\prime \prime}(t)+a(t) v(t)=g(u(t)), \\
u(0)=u(T), \quad u^{\prime}(0)=u^{\prime}(T), \quad v(0)=v(T), \quad v^{\prime}(0)=v^{\prime}(T)
\end{array}\right.
$$

where $a \in L^{1}(0, T), f:[0, T] \times(0, \infty) \rightarrow(0, \infty)$ is continuous and may be singular near the zero for the second argument, the perturbation $e \in \mathrm{L}^{1}((0, \mathrm{~T}), \mathbb{R})$ may be singular and sign-changing, $\mathrm{g}:[0, \infty) \rightarrow[0, \infty)$ is continuous and increasing satisfying $\lim _{x \rightarrow+\infty} g(x)=+\infty$. We shall establish some new results of the existence of solution for the positive, negative and semipositone cases of nonlinear term. The main tools are some fixed point theorems, which were discussed and used to solve various boundary value problems in $[1-3,5,6,8,9,13,18,19,24]$ and the references cited therein.

This paper is organized as follows. In Section 2, we introduce some lemmas which were discussed in [25] and then give our main results. In Section 3, the proof of our main results is presented based on Schauder's fixed point theorem.

\section{Preliminaries and main results}

Given $a \in \mathrm{L}^{1}(0, T)$, the notation $a \succ 0$ means that $\mathrm{a}(\mathrm{t}) \geqslant 0$ for all $t \in[0, T]$ and $a(t)>0$ for $t$ in a subset of positive measure. We consider the linear equation

$$
u^{\prime \prime}+a(t) u=0,
$$

with periodic conditions $u(0)=u(T), u^{\prime}(0)=u^{\prime}(T)$. By Fredholm's alternative, the nonhomogeneous equation $u^{\prime \prime}+a(t) u=h(t)$ admits a unique T-periodic solution

$$
u(t)=\int_{0}^{T} G(t, s) h(s) d s,
$$

where $G(t, s)$ is Green function of line (2.1) subject to periodic conditions $u(0)=u(T), u^{\prime}(0)=u^{\prime}(T)$.

Lemma 2.1 ([25]). If $\mathrm{a}(\mathrm{t}) \succ 0$, then $\mathrm{G}(\mathrm{t}, \mathrm{s})>0$ for all $(\mathrm{t}, \mathrm{s}) \in[0, \mathrm{~T}] \times[0, \mathrm{~T}]$. 
Remark 2.2. If $a(t) \succ 0$, then the solutions of (2.1) are oscillating, i.e., there are infinite zeroes, and in order to get the required distance between zeroes, the following best Sobolev constants will be used:

$$
K(q)=\left\{\begin{array}{l}
\frac{2 \pi}{q T^{1+\frac{1}{q}}}\left(\frac{2}{2+q}\right)^{1-\frac{2}{q}}\left(\frac{\Gamma\left(\frac{1}{q}\right)}{\Gamma\left(\frac{1}{2}+\frac{1}{q}\right)}\right)^{2}, \text { if } 1 \leqslant q<\infty, \\
\frac{4}{T}, \quad \text { if } q=\infty,
\end{array}\right.
$$

where $\Gamma$ is the Gamma function. For a given $p$, let us define

$$
\left\{\begin{array}{l}
p^{*}=\frac{p}{p-1}, \quad \text { if } 1 \leqslant q<\infty, \\
p^{*}=1, \quad \text { if } q=\infty
\end{array}\right.
$$

Lemma 2.3 ([25]). Assume that $\mathrm{a}(\mathrm{t}) \succ 0$, and $\mathrm{a} \in \mathrm{L}^{\mathrm{p}}(0, \mathrm{~T})$ for some $1 \leqslant \mathrm{p} \leqslant \infty$. If $\|\mathrm{a}\|_{\mathrm{p}} \leqslant \mathrm{K}\left(2 \mathrm{p}^{*}\right)$, then $\mathrm{G}(\mathrm{t}, \mathrm{s})>0$ for all $(\mathrm{t}, \mathrm{s}) \in[0, \mathrm{~T}] \times[0, \mathrm{~T}]$. Moreover, if $\mathrm{u}, v$ are the solutions of the linear equation (2.1) with initial conditions $\mathrm{u}(0)=0, \mathrm{u}^{\prime}(0)=1, v(\mathrm{~T})=0, v^{\prime}(\mathrm{T})=-1$, then $0<\mathrm{m}<\mathrm{M}$ and

$$
m:=\min _{0 \leqslant t, s \leqslant T} G(t, s)=\frac{v(0)}{2+v^{\prime}(0)-u^{\prime}(T)}, \quad M:=\max _{0 \leqslant t, s \leqslant T} G(t, s)=\frac{\max _{t}(u(t)+v(t))}{2+v^{\prime}(0)-u^{\prime}(T)} .
$$

Remark 2.4. If $\mathrm{a}(\mathrm{t}) \equiv \mathrm{k}^{2}<\left(\frac{\pi}{\mathrm{T}}\right)^{2}$, we have

$$
m=\frac{1}{2 k} \cot \left(\frac{k T}{2}\right), \quad M=\frac{1}{2 k \sin \left(\frac{k T}{2}\right)} .
$$

Now we define a set of functions

$$
\Lambda=\left\{a \in \mathrm{L}^{1}(0, \mathrm{~T}): \mathrm{a} \succ 0,\|\mathrm{a}\|_{p} \leqslant \mathrm{~K}\left(2 \mathrm{p}^{*}\right) \text { for } 1 \leqslant p \leqslant \infty\right\},
$$

and list the following assumptions:

(H0) $a \in \Lambda$.

(H1) There exist two functions $\mu_{1}, \mu_{2} \in \mathrm{C}^{+}[0, \mathrm{~T}]$, which do not vanish identically on any subinterval of $(0, T)$, and a constant $\epsilon \in(0,1)$ such that

$$
\mu_{1}(s) x^{-\epsilon} \leqslant f(s, x) \leqslant \mu_{2}(s) x^{-\epsilon}, \quad(s, x) \in(0, T) \times(0, \infty) .
$$

(H2) $\lim _{x \rightarrow+\infty} \mathrm{g}(\mathrm{x})=+\infty$.

Remark 2.5. In [4], Cao and Jiang studied the existence of periodic solutions for a system including two forcing perturbed terms $e_{i}(t) \in C[0, T], i=1,2$ with nonlinear terms being bounded and $f(+\infty)=0$. In our work, we study another case where nonlinear terms can be unbounded since forcing perturbed term $e(t) \in \mathrm{L}^{1}[0, T]$, that is, $e(t)$ can be singular in any zero measure set of $[0, T]$. On the other hand, nonlinear terms $g$ allows to be $g(+\infty)=+\infty$, which is not studied by [4].

Next we define the function $\omega: R \rightarrow R$ by

$$
\omega(t)=\int_{0}^{T} G(t, s) e(s) d s,
$$

which is the unique solution of the following problem

$$
\left\{\begin{array}{l}
x^{\prime \prime}(t)+a(t) x(t)=e(t), \quad t \in(0, T), \\
x(0)=x(T), \quad x^{\prime}(0)=x^{\prime}(T) .
\end{array}\right.
$$


Similar to (2.5), we introduce the following notations

$$
\sigma_{1}(t)=\int_{0}^{T} G(t, s) \mu_{1}(s) d s, \quad \sigma_{2}(t)=\int_{0}^{T} G(t, s) \mu_{2}(s) d s,
$$

and denote

$$
\begin{array}{ll}
\omega_{*}=\inf _{0 \leqslant t \leqslant T} \omega(t), & \omega^{*}=\sup _{0 \leqslant t \leqslant T} \omega(t), \\
\sigma_{1_{*}}=\min _{0 \leqslant t \leqslant T} \sigma_{1}(t), & \sigma_{1}^{*}=\max _{0 \leqslant t \leqslant T} \sigma_{1}(t), \\
\sigma_{2_{*}}=\min _{0 \leqslant t \leqslant T} \sigma_{2}(t), & \sigma_{2}^{*}=\max _{0 \leqslant t \leqslant T} \sigma_{2}(t) .
\end{array}
$$

By (H0)-(H1) and Lemma 2.1, we get that $\sigma_{i}^{*}, \sigma_{i *}>0, i=1,2$.

Now we give the main results of this paper for the cases of $\omega(t)$ being positive, semipositone and negative, respectively.

Theorem 2.6 (Positive case). Assume that (H0)-(H2) hold. If $\omega_{*} \geqslant 0$, then the system (1.3) has at least one positive solution.

Theorem 2.7 (Negative case). Assume that (H0) and (H1) hold. If $\omega^{*} \leqslant 0$, and

$$
\omega_{*} \geqslant\left(\frac{\epsilon^{2} \sigma_{1_{*}}}{\sigma_{2}^{* \epsilon}(M T)^{\epsilon-\epsilon^{2}}}\right)^{\frac{1}{1-\epsilon^{2}}}\left(1-\frac{1}{\epsilon^{2}}\right),
$$

then the system (1.3) has at least one positive solution.

Theorem 2.8 (Semipositone case). Assume that (H0) and (H1) are satisfied. If $\omega_{*} \leqslant 0, \omega^{*} \geqslant 0$ and

$$
\omega_{*} \geqslant x-\frac{\sigma_{1_{*}}(\mathrm{mT})^{\epsilon^{2}} \chi^{\epsilon^{2}}}{\left[\sigma_{2}^{*}+\omega^{*}[\mathrm{mT} x]^{\epsilon}\right]^{\epsilon}(\mathrm{MT})^{\epsilon}},
$$

where $\mathrm{x}$ is the unique positive solution of the equation in Lemma 3.1, then the system (1.3) has at least one positive solution.

\section{The proof of main results}

It follows from Lemma 2.1 that $(u, v) \in C[0, T] \cap C^{2}(0, T) \times C[0, T] \cap C^{2}(0, T)$ is a solution of the system (1.3) if and only if $(u, v) \in C[0, T] \times C[0, T]$ is a solution of the following nonlinear system of integral equations

$$
\left\{\begin{array}{l}
u(t)=\int_{0}^{T} G(t, s)[f(s, v(s))+e(s)] d s \\
v(t)=\int_{0}^{T} G(t, s) g(u(s)) d s .
\end{array}\right.
$$

Obviously, the above nonlinear system of integral equations is equivalent to the following operator equation

$$
u(t)=\int_{0}^{T} G(t, s)\left[f\left(s, \int_{0}^{T} G(s, \tau) g(u(\tau)) d \tau\right)+e(s)\right] d s, \quad t \in[0, T] .
$$

Define a nonlinear operator : $\mathrm{C}[0, \mathrm{~T}] \rightarrow \mathrm{C}[0, \mathrm{~T}]$ by

$$
(F u)(t)=\int_{0}^{T} G(t, s)\left[f\left(s, \int_{0}^{T} G(s, \tau) g(u(\tau)) d \tau\right)+e(s)\right] d s, \quad t \in[0, T],
$$


then the existence of solutions to the system (1.3) is equivalent to the existence of fixed points of the nonlinear operator $F$, i.e., if $z(t)$ is a fixed point of $F$ in $C[0, T]$, then the system (1.3) has a solution $(u, v)$, which can be written by

$$
\left\{\begin{array}{l}
u(t)=z(t) \\
v(t)=\int_{0}^{T} G(t, s) g(z(s)) d s, \quad t \in[0, T] .
\end{array}\right.
$$

\subsection{The proof of Theorem 2.6}

The proof of Theorem 2.6. Choose constants $r, R$ such that $0<r<R$ being fixed later, and define a closed convex set of $\mathrm{C}^{+}[0, \mathrm{~T}]$

$$
B=\left\{u \in C^{+}[0, T]: g(r) \leqslant u(t) \leqslant g(R), \quad t \in[0, T]\right\}
$$

Clearly, the operator

$$
\begin{aligned}
(F u)(t) & =\int_{0}^{T} G(t, s)\left[f\left(s, \int_{0}^{T} G(s, \tau) g(u(\tau)) d \tau\right)+e(s)\right] d s \\
& =\int_{0}^{T} G(t, s) f\left(s, \int_{0}^{T} G(s, \tau) g(u(\tau)) d \tau\right) d s+\omega(t),
\end{aligned}
$$

is completely continuous in $\mathrm{B}$ and $\mathrm{F}(\mathrm{B}) \subseteq \mathrm{C}[0, \mathrm{~T}]$.

In the following, we will prove that $F$ maps the closed convex set $B$ into itself. Given $u \in B$, by Lemma 2.3 and (H2), we have

$$
m \operatorname{Tg}(r) \leqslant \int_{0}^{T} G(s, \tau) g(u(\tau)) d \tau \leqslant M T g(R)
$$

It follows from (3.4) and (H1) that

$$
\begin{aligned}
(F u)(t) & =\int_{0}^{T} G(t, s) f\left(s, \int_{0}^{T} G(s, \tau) g(u(\tau)) d \tau\right) d s+\omega(t) \\
& \geqslant \frac{1}{[M T g(R)]^{\epsilon}} \int_{0}^{T} G(t, s) \mu_{1}(s) d s+\omega_{*} \geqslant \frac{\sigma_{1_{*}}}{[M T g(R)]^{\epsilon}}
\end{aligned}
$$

and

$$
\begin{aligned}
(F u)(t) & =\int_{0}^{T} G(t, s) f\left(s, \int_{0}^{T} G(s, \tau) g(u(\tau)) d \tau\right) d s+\omega(t) \\
& \leqslant \frac{1}{[m T g(r)]^{\epsilon}} \int_{0}^{T} G(t, s) \mu_{2}(s) d s+\omega^{*} \leqslant \frac{\sigma_{2}^{*}}{[m T g(r)]}+\omega^{*} .
\end{aligned}
$$

Thus $(F u)(t) \in B$ if $r$ and $R$ are chosen such that

$$
\frac{\sigma_{1_{*}}}{[\operatorname{MTg}(R)]^{\epsilon}} \geqslant g(r), \quad \frac{\sigma_{2}^{*}}{[m T g(r)]^{\epsilon}}+\omega^{*} \leqslant g(R) .
$$

For this, take $g(r)=\frac{1}{g(R)}$, then it is sufficient to assure (3.7) holds if the following conditions are satisfied

$$
\frac{\sigma_{1_{*}} g^{1-\epsilon}(R)}{(M T)^{\epsilon}} \geqslant 1, \quad \frac{\sigma_{2}^{*}}{[m T]^{\epsilon} g^{1-\epsilon}(R)}+\frac{\omega^{*}}{g(R)} \leqslant 1 .
$$

Noticing $\sigma_{1_{*}}>0,0<\epsilon<1$ and (H2), we have

$$
\lim _{R \rightarrow+\infty} \frac{\sigma_{1_{*}} g^{1-\epsilon}(R)}{(M T)^{\epsilon}}=+\infty, \quad \lim _{R \rightarrow+\infty}\left(\frac{\sigma_{2}^{*}}{[m T]^{\epsilon} g^{1-\epsilon}(R)}+\frac{\omega^{*}}{g(R)}\right)=0,
$$


which implies that there exists $R>0$ lager enough such that (3.8) holds, and then $F$ maps the closed convex set B into itself.

By using the Schauder's fixed point theorem, $\mathrm{F}$ has a fixed point $z \in \mathrm{B}$, and hence the system (1.3) has at least one positive solution.

\subsection{The proof of Theorem 2.7}

The proof of Theorem 2.7. In this case, in order to show that $\mathrm{F}: \mathrm{B} \rightarrow \mathrm{B}$, it is sufficient to look for some $0<\mathrm{r}<\mathrm{R}$ such that

$$
\frac{\sigma_{1_{*}}}{[M T g(R)]^{\epsilon}}+\omega_{*} \geqslant g(r), \quad \frac{\sigma_{2}^{*}}{[m T g(r)]^{\epsilon}} \leqslant g(R) .
$$

By fixing $g(R)=\frac{\sigma_{2}^{*}}{\left[m \operatorname{mos}^{\mathrm{r}}(\mathrm{r})\right]^{\epsilon}}$, then the above inequalities hold if we can find some $r$ such that

$$
0<g(r)<\left(\frac{\sigma_{2}^{*}}{(m T)^{\epsilon}}\right)^{\frac{1}{1+\epsilon}} \text { and } \omega_{*} \geqslant g(r)-\frac{\sigma_{1_{*}}}{\sigma_{2}^{* \epsilon}(M T)^{\epsilon-\epsilon^{2}}} g^{\epsilon^{2}}(r) .
$$

Obviously, the function

$$
h(x)=x-\frac{\sigma_{1_{*}}}{\sigma_{2}^{* \epsilon}(M T)^{\epsilon-\epsilon^{2}}} x^{\epsilon^{2}}
$$

possesses a minimum at

$$
x=\left(\frac{\epsilon^{2} \sigma_{1_{*}}}{\sigma_{2}^{* \epsilon}(M T)^{\epsilon-\epsilon^{2}}}\right)^{\frac{1}{1-\epsilon^{2}}}
$$

Since $\sigma_{1_{*}} \leqslant \sigma_{2}^{*}, 0<\epsilon^{2}<1$, we have

$$
x=\left(\frac{\epsilon^{2} \sigma_{1_{*}}}{\sigma_{2}^{* \epsilon}(M T)^{\epsilon-\epsilon^{2}}}\right)^{\frac{1}{1-\epsilon^{2}}}<\left(\frac{\sigma_{2}^{*}}{\sigma_{2}^{* \epsilon}}(M T)^{\epsilon-\epsilon^{2}}\right)^{\frac{1}{1-\epsilon^{2}}}=\left(\frac{\sigma_{2}^{*}}{(m T)^{\epsilon}}\right)^{\frac{1}{1+\epsilon}} .
$$

Taking $r=g^{-1}(x)$, then (3.10) holds if

$$
\omega_{*} \geqslant h(g(r))=\left(\frac{\epsilon^{2} \sigma_{1_{*}}}{\sigma_{2}^{* \epsilon}(M T)^{\epsilon-\epsilon^{2}}}\right)^{\frac{1}{1-\epsilon^{2}}}\left(1-\frac{1}{\epsilon^{2}}\right),
$$

which also guarantees that (3.9) holds. Consequently, by mean of Schauder's fixed point theorem, F has a fixed point $z \in B$, and hence the system (1.3) has at least one positive solution.

\subsection{The proof of Theorem 2.8}

In this section, we firstly define two positive constants as follows:

$$
A=\frac{\sigma_{2}^{*} \sigma_{1_{*}}(m T)^{\epsilon^{2}} \epsilon^{2}}{(M T)^{\epsilon}}, \quad B=\omega^{*}(m T)^{\epsilon} .
$$

In order to prove Theorem 2.8, we need the following lemma.

Lemma 3.1. Let $0<\epsilon<1$, then the equation

$$
x^{1-\epsilon^{2}}\left(\sigma_{2}^{*}+B x^{\epsilon}\right)^{1+\epsilon}=A,
$$

has a unique positive solution $\mathrm{x} \in(0, \infty)$ satisfying

$$
0<x<\lambda:=A^{\frac{1}{2+2 \epsilon}}(\mathrm{mT})^{-\frac{\epsilon}{2}} .
$$


Proof. Let $h(x)=A-x^{1-\epsilon^{2}}\left(\sigma_{2}^{*}+B x^{\epsilon}\right)^{1+\epsilon}$, then from $0<\epsilon<1, A>0, B>0$, we have

$$
h(0)=A>0, \quad h(+\infty)=-\infty .
$$

On the other hand

$$
h^{\prime}(x)=-\left(1-\epsilon^{2}\right) x^{-\epsilon^{2}}\left(\sigma_{2}^{*}+B x^{\epsilon}\right)^{1+\epsilon}-B(1+\epsilon) x^{\epsilon-\epsilon^{2}}\left(\sigma_{2}^{*}+B x^{\epsilon}\right)^{\epsilon}<0, \quad x \in(0, \infty) .
$$

Thus by (3.14)-(3.15) and the continuity of $h$, (3.12) has a unique positive solution $x \in(0,+\infty)$.

Now we prove (3.13) holds. In fact, noticing that $0<\epsilon<1, \sigma_{2}^{*} \geqslant \sigma_{1_{*}}, M \geqslant m$, we have

$$
\begin{aligned}
0<\lambda & =A^{\frac{1}{2+2 \epsilon}}(m T)^{-\frac{\epsilon}{2}}=\left(\frac{\sigma_{2}^{*} \sigma_{1_{*}}(m T)^{\epsilon^{2}} \epsilon^{2}}{(M T)^{\epsilon}}\right)^{\frac{1}{2+2 \epsilon}}(m T)^{-\frac{\epsilon}{2}} \leqslant \sigma_{2}^{*} \frac{1}{1+\epsilon}\left(\frac{(m T)^{\epsilon^{2}}}{(m T)^{\epsilon}}\right)^{\frac{1}{2+2 \epsilon}}(m T)^{-\frac{\epsilon}{2}} \\
& =\sigma_{2}^{*} \frac{1}{1+\epsilon}(m T)^{-\frac{\epsilon}{1+\epsilon}} .
\end{aligned}
$$

It follows from (3.16) that

$$
\begin{aligned}
h(\lambda) & =A-\lambda^{1-\epsilon^{2}}\left(\sigma_{2}^{*}+B \lambda^{\epsilon}\right)^{1+\epsilon}=\lambda^{2+2 \epsilon}(m T)^{\epsilon(1+\epsilon)}-\lambda^{1-\epsilon^{2}}\left(\sigma_{2}^{*}+\omega^{*} \lambda^{\epsilon}\right)^{1+\epsilon} \\
& =\lambda^{1-\epsilon^{2}}\left[\lambda^{(1+\epsilon)^{2}}(m T)^{\epsilon(1+\epsilon)}-\left(\sigma_{2}^{*}+\omega^{*} \lambda^{\epsilon}\right)^{1+\epsilon}\right] \\
& <\lambda^{1-\epsilon^{2}}\left[\lambda^{(1+\epsilon)^{2}}(m T)^{\epsilon(1+\epsilon)}-\sigma_{2}^{* 1+\epsilon}\right]<0 .
\end{aligned}
$$

Since $h(0)>0$, the unique solution of the equation $(3.12)$ is in $(0, \lambda)$.

The proof of Theorem 2.8. We follow the same strategy and notations as in the proof of Theorems 2.6-2.7. In this case, to prove $T: B \rightarrow B$, it is sufficient to find some $r$ and $R$ such that $0<r<R$ and

$$
\frac{\sigma_{1_{*}}}{[\operatorname{MTg}(R)]^{\epsilon}}+\omega_{*} \geqslant g(r), \quad \frac{\sigma_{2}^{*}}{[m \operatorname{Tg}(r)]^{\epsilon}}+\omega^{*} \leqslant g(R) .
$$

If we fix $g(R)=\frac{\sigma_{2}^{*}}{\left[m \operatorname{mg}^{\prime}(r)\right]^{\epsilon}}+\omega^{*}$, then the inequalities of (3.17) hold if $r$ satisfies

$$
\frac{\sigma_{2}^{*}}{[m \operatorname{Tg}(r)]^{\epsilon}}+\omega^{*}>g(r)
$$

and

$$
\omega_{*} \geqslant g(r)-\frac{\sigma_{1_{*}}}{[M T g(R)]^{\epsilon}}=g(r)-\frac{\sigma_{1_{*}}(m T) \epsilon^{\epsilon^{2}} g^{\epsilon^{2}(r)}}{\left[\sigma_{2}^{*}+\omega^{*}[m T g(r)]^{\epsilon}\right]^{\epsilon}(M T)^{\epsilon}}
$$

Let

$$
\varphi(x)=x-\frac{\sigma_{1_{*}}(\mathrm{mT}) \epsilon^{\epsilon^{2}} \chi^{\epsilon^{2}}}{\left[\sigma_{2}^{*}+\omega^{*}[\mathrm{mT} x]^{\epsilon}\right]^{\epsilon}(\mathrm{MT})^{\epsilon}}
$$

and then

$$
\varphi^{\prime}(x)=1-\frac{\sigma_{2}^{*} \sigma_{1_{*}}(\mathrm{mT}) \epsilon^{\epsilon^{2}} \epsilon^{2} \chi^{\epsilon^{2}-1}}{\left[\sigma_{2}^{*}+\omega^{*}[\mathrm{mT} x]^{\epsilon}\right]^{\epsilon+1}(\mathrm{MT})^{\epsilon}}=1-\frac{A x^{\epsilon^{2}-1}}{\left(\sigma_{2}^{*}+B x^{\epsilon}\right)^{\epsilon+1}},
$$

we have $\varphi^{\prime}(0)=-\infty, \varphi^{\prime}(\infty)=1$, and consequently there exists $x$ such that $\varphi^{\prime}(x)=0$, that is, $x$ is the unique solution of the equation $x^{1-\epsilon^{2}}\left(\sigma_{2}^{*}+B x^{\epsilon}\right)^{1+\epsilon}=A$. Moreover

$$
\varphi^{\prime \prime}(x)=\frac{A\left(1-\epsilon^{2}\right) x^{\epsilon^{2}-2}}{\left(\sigma_{2}^{*}+B x^{\epsilon}\right)^{\epsilon+1}}+\frac{A \epsilon(1+\epsilon) x^{\epsilon^{2}+\epsilon-2}}{\left(\sigma_{2}^{*}+B x^{\epsilon}\right)^{\epsilon+2}}>0,
$$


which implies that $\varphi(x)$ possesses a minimum at $x$, i.e., $\varphi(x)=\min _{x \in(0, \infty)} \varphi(x)$. Taking $r=g^{-1}(x)$, then (3.18) holds if (2.7) is satisfied. Since

Now we prove that the inequality $R>r$ also holds. To do this, we only need to prove $g(R)>g(r)$.

$$
g(R)=\frac{\sigma_{2}^{*}}{[m \operatorname{Tg}(r)]^{\epsilon}}+\omega^{*},
$$

and $g(r)=x$ satisfies the equation (3.12), we have $g^{1-\epsilon^{2}}(r)\left(\sigma_{2}^{*}+B g^{\epsilon}(r)\right)^{1+\epsilon}=A$, i.e.,

$$
g^{1+\epsilon}(r) g^{1+\epsilon}(R)=A(m T)^{-\epsilon(1+\epsilon)}
$$

It follows from Lemma 3.1 that

$$
0<\mathrm{g}(\mathrm{r})<\mathrm{A}^{\frac{1}{2+2 \epsilon}}(\mathrm{mT})^{-\frac{\epsilon}{2}}
$$

From (3.19) and (3.20), we have

$$
g(R)=\frac{1}{g(r)}\left(A(m T)^{-\epsilon(1+\epsilon)}\right)^{\frac{1}{1+\epsilon}}>A^{\frac{1}{2+2 \epsilon}}(m T)^{-\frac{\epsilon}{2}}>g(r) .
$$

Hence (2.7) guarantees (3.17) holds, and thus Schauder's fixed point theorem implies that the system (1.3) has at least one positive solution.

\section{Numerical examples}

In this section, we take $p=2, T=1, a(t) \equiv k^{2}=\frac{1}{4}$, then $a(t)=\frac{1}{4}<\left(\frac{\pi}{T}\right)^{2}=\pi^{2}$ and

$$
\|\mathrm{a}\|_{2}=0.25 \leqslant \mathrm{~K}\left(2 \mathrm{p}^{*}\right)=\mathrm{K}(4)=\frac{\pi}{2}\left(\frac{1}{3}\right)^{\frac{1}{2}}\left(\frac{\Gamma\left(\frac{1}{4}\right)}{\Gamma\left(\frac{3}{4}\right)}\right)^{2}=7.9398,
$$

that is $a \in \Lambda$, and (H0) holds. Moreover,

$$
\mathrm{m}=\frac{1}{2 \mathrm{k}} \cot \left(\frac{\mathrm{kT}}{2}\right)=\cot \left(\frac{1}{4}\right), \quad M=\frac{1}{\sin \left(\frac{1}{4}\right)} .
$$

We consider the following singular periodic differential system

$$
\left\{\begin{array}{l}
u^{\prime \prime}(t)+u(t)=\frac{3}{2 v^{\frac{1}{2}}(t)}+e(t), \quad v^{\prime \prime}(t)+v(t)=u^{2}(t) \\
u(0)=u(1), \quad u^{\prime}(0)=u^{\prime}(1), \quad v(0)=v(1), \quad v^{\prime}(0)=v^{\prime}(1)
\end{array}\right.
$$

Example 4.1. If $e(t)=t^{-\frac{1}{2}}$, let $f(t, x)=\frac{3}{2} x^{-\frac{1}{2}}, g(y)=y^{2}, \mu_{1}(t)=1, \mu_{2}(t)=2, \epsilon=\frac{1}{2}$, and then

$$
\mu_{1}(t) x^{-\frac{1}{2}} \leqslant f(t, x)=\frac{3}{2} x^{-\frac{1}{2}} \leqslant \mu_{2}(t) x^{-\frac{1}{2}}, \quad x \in(0,+\infty), \quad \lim _{y \rightarrow+\infty} y^{2}=+\infty,
$$

which imply that (H1) and (H2) hold.

On the other hand,

$$
\omega_{*}=\inf _{0 \leqslant t \leqslant 1} \omega(t)=\inf _{0 \leqslant t \leqslant 1} \int_{0}^{1} G(t, s) s^{-\frac{1}{2}} d s=2 \cot \left(\frac{1}{4}\right) \geqslant 0 .
$$

According to Theorem 2.6, the singular periodic differential system (4.2) has at least one positive solution. 
Example 4.2. If $e(t)=-\frac{t^{-\frac{1}{2}}}{100}$, then the system (4.2) reduces to the system (1.1). Still let $f(t, x)=$ $\frac{3}{2} x^{-\frac{1}{2}}, g(y)=y^{2}, \mu_{1}(t)=1, \mu_{2}(t)=2, \epsilon=\frac{1}{2}$, by Example 4.1, we have (H1) holds.

Now we compute $\omega_{*}$ and $\omega^{*}$. Clearly $\omega^{*}<0$, and

$$
\omega_{*}=\inf _{0 \leqslant t \leqslant 1} \omega(t)=-\inf _{0 \leqslant t \leqslant 1} \int_{0}^{1} G(t, s) \frac{s^{-\frac{1}{2}}}{100} d s=-\frac{2}{100 \sin \left(\frac{1}{4}\right)} \approx-0.08084 .
$$

But,

so we have

$$
\left(\frac{\epsilon^{2} \sigma_{1_{*}}}{\sigma_{2}^{* \epsilon}(M T)^{\epsilon-\epsilon^{2}}}\right)^{\frac{1}{1-\epsilon^{2}}}\left(1-\frac{1}{\epsilon^{2}}\right)=-3\left[\frac{\mathrm{m}}{4 \sqrt{2} \mathrm{M}^{\frac{3}{4}}}\right]^{\frac{4}{3}} \approx-0.3225
$$

$$
\omega_{*} \geqslant\left(\frac{\epsilon^{2} \sigma_{1_{*}}}{\sigma_{2}^{* \epsilon}(M T)^{\epsilon-\epsilon^{2}}}\right)^{\frac{1}{1-\epsilon^{2}}}\left(1-\frac{1}{\epsilon^{2}}\right) .
$$

Thus according to Theorem 2.7, the singular periodic differential system (4.2) has at least one positive solution.

Example 4.3. Take

$$
e(t)= \begin{cases}-\frac{t^{-\frac{1}{2}}}{100}, & 0 \leqslant t \leqslant \frac{1}{2}, \\ \frac{t^{-\frac{1}{2}}}{42}, & \frac{1}{2}<t \leqslant 1,\end{cases}
$$

and still let $f(t, x)=\frac{3}{2} x^{-\frac{1}{2}}, g(y)=y^{2}, \mu_{1}(t)=1, \mu_{2}(t)=2, \epsilon=\frac{1}{2}$, then (Ho) and (H1) hold.

In the following, we verify

$$
\omega_{*} \geqslant x-\frac{\sigma_{1_{*}}(\mathrm{mT})^{\epsilon^{2}} \chi^{\epsilon^{2}}}{\left[\sigma_{2}^{*}+\omega^{*}[\mathrm{mT} x]^{\epsilon}\right]^{\epsilon}(\mathrm{MT})^{\epsilon}}
$$

holds. Firstly, we have

$$
\begin{aligned}
& \omega_{*}=\inf _{0 \leqslant t \leqslant 1} \omega(t)=\inf _{0 \leqslant t \leqslant 1}\left(-\int_{0}^{\frac{1}{2}} G(t, s) \frac{s^{-\frac{1}{2}}}{100} d s+\int_{\frac{1}{2}}^{1} G(t, s) \frac{s^{-\frac{1}{2}}}{42} d s\right) \approx-0.0026, \\
& \omega^{*}=\sup _{0 \leqslant t \leqslant 1} \omega(t)=\inf _{0 \leqslant t \leqslant 1}\left(-\int_{0}^{\frac{1}{2}} G(t, s) \frac{s^{-\frac{1}{2}}}{100} d s+\int_{\frac{1}{2}}^{1} G(t, s) \frac{s^{-\frac{1}{2}}}{42} d s\right) \approx 0.001, \\
& \sigma_{1_{*}}=\min _{0 \leqslant t \leqslant T} \sigma_{1}(t)=\min _{0 \leqslant t \leqslant T} \int_{0}^{1} G(t, s) \mu_{1}(s) d s \approx 3.9163, \\
& \sigma_{2^{*}}=\max _{0 \leqslant t \leqslant T} \sigma_{2}(t)=\max _{0 \leqslant t \leqslant T} \int_{0}^{1} G(t, s) \mu_{2}(s) d s \approx 8.0839 .
\end{aligned}
$$

On the other hand, the equation $x^{1-\epsilon^{2}}\left(\sigma_{2}^{*}+B x^{\epsilon}\right)^{1+\epsilon}=A$, i.e., $x^{\frac{3}{4}}\left(8.0839+0.002 x^{\frac{1}{2}}\right)^{\frac{5}{4}}=5.5381$ has unique solution $x=0.301$. Thus

$$
x-\frac{\sigma_{1_{*}}(\mathrm{mT})^{\epsilon^{2}} \chi^{\epsilon^{2}}}{\left[\sigma_{2}^{*}+\omega^{*}[\mathrm{mT} x]^{\epsilon}\right]^{\epsilon}(\mathrm{MT})^{\epsilon}}=0.301-\frac{3.9163 \times\left(0.301 \cot \frac{1}{4}\right)^{\frac{1}{4}} \times\left(\sin \frac{1}{4}\right)^{\frac{1}{2}}}{\left(8.0839+0.001 \times\left(0.301 \cot \frac{1}{4}\right)^{\frac{1}{2}}\right)^{\frac{1}{2}}} \approx-0.4128,
$$

so $\omega_{*} \approx-0.0026>-0.4128$. According to Theorem 2.8 , the singular periodic differential system (4.2) has at least one positive solution. 


\section{Acknowledgment}

The authors were supported financially by the National Natural Science Foundation of China (11571296 11371221), the Youth Project of MOE (Ministry of Education in China) Humanity and Social Science Foundation (16YJC630101), the Youth Project of Hunan Provincial Philosophy and Social Science Foundation (15YBA407), the Foundation of the Excellent Youth Scholars of Hunan Provincial Department of Education (14B188) and the Natural Science Foundation of Shandong Province of China (ZR2014AM009).

\section{References}

[1] Z.-B. Bai, Solvability for a class of fractional m-point boundary value problem at resonance, Comput. Math. Appl., 62 (2011), 1292-1302. 1

[2] Z.-B. Bai, Eigenvalue intervals for a class of fractional boundary value problem, Comput. Math. Appl., 64 (2012), $3253-$ 3257.

[3] Z.-B. Bai, Y.-H. Zhang, The existence of solutions for a fractional multi-point boundary value problem, Comput. Math. Appl., 60 (2010), 2364-2372. 1

[4] Z.-W. Cao, D.-Q. Jiang, Periodic solutions of second order singular coupled systems, Nonlinear Anal., 71 (2009), 36613667. 1, 2.5

[5] Y.-J. Cui, Computation of topological degree in ordered Banach spaces with lattice structure and applications, Appl. Math., 58 (2013), 689-702. 1

[6] Y.-J. Cui, Uniqueness of solution for boundary value problems for fractional differential equations, Appl. Math. Lett., 51 (2016), 48-54. 1

[7] Y.-J. Cui, L.-S. Liu, X.-Q. Zhang, Uniqueness and existence of positive solutions for singular differential systems with coupled integral boundary value problems, Abstr. Appl. Anal., 2013 (2013), 9 pages. 1

[8] Y.-J. Cui, J.-X. Sun, Fixed point theorems for a class of nonlinear operators in Hilbert spaces and applications, Positivity, 15 (2011), 455-464. 1

[9] Y.-J. Cui, J.-X. Sun, Fixed point theorems for a class of nonlinear operators in Hilbert spaces with lattice structure and application, Fixed Point Theory Appl., 2013 (2013), 9 pages. 1

[10] Y.-J. Cui, J.-X. Sun, Y.-M. Zou, Global bifurcation and multiple results for Sturm-Liouville problems, J. Comput. Appl. Math., 235 (2011), 2185-2192. 1

[11] Y.-J. Cui, Y.-M. Zou, Existence of solutions for second-order integral boundary value problems, Nonlinear Analysis: Modelling and Control, 21 (2016), 828-838. 1

[12] Y.-J. Cui, Y.-M. Zou, An existence and uniqueness theorem for a second order nonlinear system with coupled integral boundary value conditions, Appl. Math. Comput., 256 (2015), 438-444. 1

[13] Y.-J. Cui, Solvability of second-order boundary-value problems at resonance involving integral conditions, Electronic Journal of Differential Equations, 2012 (2012), 1-9. 1

[14] A. Demir, A. Mehrotra, J. Roychowdhury, Phase noise in oscillators: A unifying theory and numerical methods for characterization, IEEE Trans. Circuits Syst. Fundam. Theory Appl., 47 (2000), 655-674. 1

[15] C. W. Gardiner, Handbook of stochastic methods for physics, chemistry and the natural sciences, Third edition, Springer Series in Synergetics, Springer-Verlag, Berlin, (2004). 1

[16] X.-A. Hao, L.-S. Liu, Y.-H. Wu, Existence and multiplicity results for nonlinear periodic boundary value problems, Nonlinear Anal., 72 (2010), 3635-3642. 1

[17] V. G. Ivancevic, T. T. Ivancevic, Geometrical dynamics of complex systems: a unified modelling approach to physics, control, biomechanics, neurodynamics and psycho-socio-economical dynamics, Springer Science \& Business Media, (2006). 1

[18] H.-Y. Li, J.-X. Sun, Positive solutions of superlinear semipositone nonlinear boundary value problems, Comput. Math. Appl., 61 (2011), 2806-2815. 1

[19] H.-Y. Li, F. Sun, Existence of solutions for integral boundary value problems of second-order ordinary differential equations, Bound. Value Probl., 2012 (2012), 7 pages. 1

[20] Y. Loya, Recolonization of Red Sea corals affected by natural catastrophes and manmade perturbations, Ecol., 57 (1976), 278-289. 1

[21] W. Moon, J. S. Wettlaufer, A stochastic perturbation theory for non-autonomous systems, J. Math. Phys., 54 (2013), 31 pages. 1

[22] D.-B. Qian, L. Chen, X.-Y. Sun, Periodic solutions of superlinear impulsive differential equations: a geometric approach, J. Differential Equations, 258 (2015), 3088-3106. 1

[23] S.-T. Qin, X.-P. Xue, Periodic solutions for nonlinear differential inclusions with multivalued perturbations, J. Math. Anal. Appl., 424 (2015), 998-1005. 1

[24] J.-X. Sun, Y.-J. Cui, Fixed point theorems for a class of nonlinear operators in Riesz spaces, Fixed Point Theory, 14 (2013), 185-192. 1

[25] P. J. Torres, Existence of one-signed periodic solutions of some second-order differential equations via a Krasnoselskii fixed point theorem, J. Differential Equations, 190 (2003), 643-662. 1, 1, 2.1, 2.3 
[26] J. R. Ward, Jr., Periodic solutions of ordinary differential equations with bounded nonlinearities, Topol. Methods Nonlinear Anal., 19 (2002), 275-282. 1, 1

[27] X.-G. Zhang, L.-S. Liu, Y.-H. Wu, Existence results for multiple positive solutions of nonlinear higher order perturbed fractional differential equations with derivatives, Appl. Math. Comput., 219 (2012), 1420-1433.

[28] X.-G. Zhang, L.-S. Liu, Y.-H. Wu, Multiple positive solutions of a singular fractional differential equation with negatively perturbed term, Math. Comput. Modelling, 55 (2012), 1263-1274.

[29] X.-G. Zhang, Y.-H. Wu, L. Caccetta, Nonlocal fractional order differential equations with changing-sign singular perturbation, Appl. Math. Modelling, 39 (2015), 6543-6552. 1 